\title{
LMI OPTIMIZATION PROBLEM OF DELAY-DEPENDENT ROBUST STABILITY CRITERIA FOR STOCHASTIC SYSTEMS WITH POLYTOPIC AND LINEAR FRACTIONAL UNCERTAINTIES
}

\author{
PAgAVATHigounder BALASUBRAMANIAM *, SHANMUGAM LAKSHMANAN *, \\ RAJAN RAKKIYAPPAN ** \\ ${ }^{*}$ Department of Mathematics \\ Gandhigram Rural Institute-Deemed University, Gandhigram 624 302, Tamilnadu, India \\ e-mail: balugru@gmail.com \\ ${ }^{* *}$ Department of Mathematics \\ Bharathiar University, Coimbatore 641 046, Tamilnadu, India
}

\begin{abstract}
This paper studies an LMI optimization problem of delay-dependent robust stability criteria for stochastic systems with polytopic and linear fractional uncertainties. The delay is assumed to be time-varying and belong to a given interval, which means that lower and upper bounds of this interval time-varying delay are available. The uncertainty under consideration includes polytopic-type uncertainty and linear fractional norm-bounded uncertainty. Based on the new Lyapunov-Krasovskii functional, some inequality techniques and stochastic stability theory, delay-dependent stability criteria are obtained in terms of Linear Matrix Inequalities (LMIs). Moreover, the derivative of time delays is allowed to take any value. Finally, four numerical examples are given to illustrate the effectiveness of the proposed method and to show an improvement over some results found in the literature.
\end{abstract}

Keywords: delay-dependent stability, linear matrix inequality, Lyapunov-Krasovskii functional, stochastic systems.

\section{Introduction}

Time-delay occurs in many dynamical systems such as biological systems, chemical, metallurgical processing and nuclear reactors, long-transmission lines in pneumatic and hydraulic systems as well as electrical networks (Kolmanoskii and Myshkis, 1992). Frequently, it has been a source of the oscillations, instability and poor performance. Considerable effort has been applied to different aspects of linear time-delay systems in recent years (Hale and Verduyn lunel, 1993; Huang and Zhou, 2000; Ivanesu et al., 2000; Mahmoud and AlMuthairi, 1994; Liu, 2005; Xue and Qiu, 2000; Xia and Jia, 2003). Moreover, the stability analysis of interval time-varying systems has been focused on as a topic of theoretical and practical importance (He et al., 2006; Jiang and Han, 2008; Yue, 2006; Jiang and Han, 2006; Kwon and Park, 2008). Systems with interval time-varying delays mean that the lower bound of the time delay which guarantees the stability of the system is not restricted to zero. A typical example of dynamical systems with in- terval time-varying delays is a networked control system (Yue, 2006).

Uncertainties are frequently encountered in various engineering and communication systems. The characteristics of dynamic systems are significantly affected by the presence of uncertainties, even to the extent of instability in an extreme situation (Zhou et al., 2006).

In engineering applications, it is very common that one does not know exactly the system under investigation; that is, the system contains some elements (blocks) that are uncertain. Usually it is known that these uncertain elements belong to some specific admissible domains, which in turn depend on the nature of the elements and also on the information available about the system. In other words, it is known only that the system belongs to the family of systems that arises when the uncertain elements (blocks) range over the admissible domains and therefore one may treat the family as a new object for analysis. This family is referred to as an uncertain system. When it is possible to show that all systems of the family are stable, the stability of the original system that is a particular 
member of the family is guaranteed. The robust stability problem considers the stability problem of systems that contain some uncertainties.

Over the past decade, much effort has been spent on the analysis and synthesis of uncertain systems with timedelay (see, e.g., the works of Chen (2002) Kim (2001), Liu and Zhang (2005) and the references therein).

In this paper, the stability analysis for stochastic system is investigated under polytopic type uncertainty and linear fractional uncertainty. First, polytopic uncertainties can arise when the uncertain matrix in norm-bounded uncertainties provides some prior known structures of uncertainties. Therefore the polytopic type uncertainty can be regarded as an important class of parameter uncertainty. Recently, the problem of robust stability and stabilization for delayed systems with polytopic uncertainties have been studied and LMI-based approaches have been developed (Li et al., 2008; Xia and Jia, 2002; He et al., 2004; Geromel and Colaneri, 2006; Chesi et al., 2007; Ramos and Peres, 2001; Xu et al., 2004). As is well known, usually fractional uncertainties are more general than norm-bounded uncertainties. Recently, some results on the stability of systems with linear fractional uncertainty have been reported (Li et al., 2007; Balasubramaniam et al., 2009; Gu et al., 2003; Balasubramaniam and Lakshmanan, 2011).

In recent years, increasing efforts have been made to study stochastic systems with time-delays. The stability and control problem for uncertain stochastic delayed systems has been extensively investigated by a considerable number of researchers (Miyamura and Aihara, 2004; Yan et al., 2009; Zhang et al., 2009; 2008; Chen et al., 2005; Yue and Han, 2005; He et al., 2010). Tian et al. (2010), dealt with the problem of robust $H_{\infty}$ control design for nonlinear networked control systems, which are presented in the form of a T-S fuzzy model with a time-varying input delay and the whole variation interval of the delay is divided into two subintervals of equal length, which is different from the existing method. Recently, only few authors have discussed stability criteria for a system with linear fractional and polytopic uncertainties.

Based on the above motivations, this paper aims to develop an LMI optimization problem of delay-dependent robust stability criteria for stochastic systems by constructing a new Lyapunov-Krasovskii functional with integral terms involving lower and upper bounds of interval time-varying delays such as

$$
\begin{gathered}
\int_{t-\frac{\tau_{m}}{2}}^{t}\left[\begin{array}{c}
x(s) \\
x\left(s-\frac{\tau_{m}}{2}\right)
\end{array}\right]^{T}\left[\begin{array}{ll}
Q_{1} & Q_{2} \\
Q_{2}^{T} & Q_{3}
\end{array}\right]\left[\begin{array}{c}
x(s) \\
x\left(s-\frac{\tau_{m}}{2}\right)
\end{array}\right] \mathrm{d} s, \\
\int_{t-\frac{\tau_{M}}{2}}^{t}\left[\begin{array}{c}
x(s) \\
x\left(s-\frac{\tau_{M}}{2}\right)
\end{array}\right]^{T}\left[\begin{array}{ll}
Q_{4} & Q_{5} \\
Q_{5}^{T} & Q_{6}
\end{array}\right]\left[\begin{array}{c}
x(s) \\
x\left(s-\frac{\tau_{M}}{2}\right)
\end{array}\right] \mathrm{d} s,
\end{gathered}
$$

$$
\begin{aligned}
& \frac{\tau_{m}}{2} \int_{-\frac{\tau_{m}}{2}}^{0} \mathrm{~d} s \int_{t+s}^{t} y^{T}(\theta) R_{4} y(\theta) \mathrm{d} \theta, \\
& \frac{\tau_{M}}{2} \int_{-\frac{\tau_{M}}{2}}^{0} \mathrm{~d} s \int_{t+s}^{t} y^{T}(\theta) R_{5} y(\theta) \mathrm{d} \theta, \\
& \delta \int_{-\tau_{M}}^{-\tau_{m}} \mathrm{~d} s \int_{t+s}^{t} y^{T}(\theta) R_{6} y(\theta) \mathrm{d} \theta .
\end{aligned}
$$

by employing some analytical techniques, sufficient conditions are derived for the stochastic systems considered in terms of LMIs, which can be easily calculated by the MATLAB LMI Control Toolbox. Moreover, a polytopictype and linear fractional uncertainty which includes as a norm-bounded uncertainty as a special case is discussed. Some numerical examples are given to illustrate the effectiveness and conservativeness of the proposed method.

Notation. Throughout this paper, $\mathbb{R}^{n}$ and $\mathbb{R}^{n \times n}$ denote, respectively, the $n$-dimensional Euclidean space and the set of all $n \times n$ real matrices. The superscript $T$ denotes the transposition and the notation $X \geq Y$ (respectively $X>Y$ ), where $X$ and $Y$ are symmetric matrices, means that $X-Y$ is positive semi-definite (respectively positive definite). $I_{n}$ is the $n \times n$ identity matrix. $|\cdot|$ is the Euclidean norm in $\mathbb{R}^{n}$. Moreover, let $(\Omega, \mathcal{F}, \mathcal{P})$ be a complete probability space with a filtration $\left\{\mathcal{F}_{t}\right\}_{t \geq 0}$ satisfying the usual conditions (that is, the filtration contains all $\mathcal{P}$ null sets and is right continuous). The asterisk $*$ always denotes the symmetric block in one symmetric matrix. Sometimes, the arguments of a function or a matrix will be omitted in the analysis when no confusion can arise.

\section{Problem description and preliminaries}

Consider the following stochastic system with state delay:

$$
\begin{aligned}
\mathrm{d} x(t)= & {[A(t) x(t)+B(t) x(t-\tau(t))] \mathrm{d} t } \\
& +[C(t) x(t)+D(t) x(t-\tau(t))] \mathrm{d} w(t), \\
x(t)= & \phi(t), \quad t \in\left[-\tau_{M}, 0\right],
\end{aligned}
$$

where $x(t) \in \mathbb{R}^{n}$ is the state vector, $A(t)=A+$ $\Delta A(t), B(t)=B+\Delta B(t), C(t)=C+\Delta C(t)$ and $D(t)=D+\Delta D(t), A, B, C$ and $D$ are known real matrices of appropriate dimensions, $\Delta A(t), \Delta B(t), \Delta C(t)$ and $\Delta D(t)$ are unknown real matrices of appropriate dimensions representing system time-varying parameter uncertainties; $\omega(t)$ denotes one dimensional Brownian motion satisfying $E\{\mathrm{~d} \omega(t)\}=0$ and $E\left\{\mathrm{~d} \omega(t)^{2}\right\}=\mathrm{d} t$. It is defined on a complete probability space $(\Omega, \mathcal{F}, \mathcal{P})$ with a natural filtration $\left\{\mathcal{F}_{t}\right\}$. Here $\phi(t)$ is any given initial data in $L_{\mathcal{F}_{0}}^{2}\left(\left[-\tau_{M}, 0\right] ; \mathbb{R}^{n}\right)$. Furthermore, $\tau(t)$ denotes the time varying bounded delay and is assumed to satisfy

$$
0 \leq \tau_{m} \leq \tau(t) \leq \tau_{M}, \quad \dot{\tau}(t) \leq \mu,
$$

where $\tau_{m}, \tau_{M}$ and $\mu$ are constants. We consider robust stability of the system described by (1) and (2) subject to 
polytopic uncertainty. For polytopic uncertainty, matrices $A, B, C$ and $D$ in (1) can be expressed as

$$
\begin{aligned}
{\left[\begin{array}{llll}
A & B & C & D
\end{array}\right] } \\
\quad=\sum_{i=1}^{r} \lambda_{i}\left[\begin{array}{llll}
A^{(i)} & B^{(i)} & C^{(i)} & D^{(i)}
\end{array}\right]
\end{aligned}
$$

where

$$
\sum_{i=1}^{r} \lambda_{i}=1, \quad 0 \leq \lambda_{i} \leq 1 .
$$

Next, we address the linear fractional norm-bounded uncertainty. Suppose that matrices $A, B, C$ and $D$ have parameter perturbations $\Delta A(t), \Delta B(t), \Delta C(t)$ and $\Delta D(t)$, which are in the form of

$$
\begin{array}{r}
{\left[\begin{array}{llll}
\Delta A(t) & \Delta B(t) & \Delta C(t) & \Delta D(t)
\end{array}\right]} \\
=H \Lambda(t)\left[\begin{array}{llll}
E & E_{1} & E_{2} & E_{3}
\end{array}\right]
\end{array}
$$

where $E, E_{1}, E_{2}$ and $E_{3}$ are given matrices. The class of parametric uncertainties $\Lambda(t)$ that satisfy

$$
\Lambda(t)=[I-F(t) J]^{-1} F(t)
$$

is said to be admissible, where $J$ is also a known matrix satisfying

$$
I-J J^{T}>0
$$

and $F(t)$ is an uncertain matrix satisfying

$$
F^{T}(t) F(t) \leq I
$$

Definition 1. The stochastic time-delay system (1) is said to be robustly stochastically stable if there exists a positive scalar $c>0$ such that for all admissible uncertainties

$$
\lim _{T \longrightarrow \infty} \mathbb{E} \int_{0}^{T} x^{T}(t) x(t) \mathrm{d} t \leq c \sup _{s \in\left[-\tau_{M}, 0\right]} \mathbb{E}\|\phi(s)\|^{2} .
$$

In obtaining the main results of this paper, the following lemmas will be essential for the proof.

Lemma 1. (Boyd et al., 1994) (Schur complement) Given constant matrices $\Omega_{1}, \Omega_{2}$ and $\Omega_{3}$ of appropriate dimensions, where $\Omega_{1}^{T}=\Omega_{1}$ and $\Omega_{2}^{T}=\Omega_{2}>0$, we have

$$
\Omega_{1}+\Omega_{3}^{T} \Omega_{2}^{-1} \Omega_{3}<0
$$

if and only if

$$
\left[\begin{array}{cc}
\Omega_{1} & \Omega_{3}^{T} \\
* & -\Omega_{2}
\end{array}\right]<0, \quad \text { or } \quad\left[\begin{array}{cc}
-\Omega_{2} & \Omega_{3} \\
* & \Omega_{1}
\end{array}\right]<0 .
$$

Lemma 2. (Gu, 2000) For any constant matrix $M>0$, any scalars $a$ and $b$ with $a<b$ and $a$ vector function $x(t):[a, b] \longrightarrow \mathbb{R}^{n}$ such that the integrals concerned are well defined, we have

$$
\begin{aligned}
{\left[\int_{a}^{b} x(s) \mathrm{d} s\right]^{T} M } & {\left[\int_{a}^{b} x(s) \mathrm{d} s\right] } \\
& \leq(b-a)\left[\int_{a}^{b} x^{T}(s) M x(s) \mathrm{d} s\right] .
\end{aligned}
$$

Lemma 3. (Li et al., 2007) Suppose $\Lambda(t)$ is given by (5)(7). Given matrices $M=M^{T}, S$ and $N$ of appropriate dimensions, the inequality

$$
M+S \Lambda(t) N+N^{T} \Lambda^{T}(t) S^{T}<0
$$

holds for $F(t)$ such that $F^{T}(t) F(t) \leq I$ if, and only if, for some $\epsilon>0$

$$
\left[\begin{array}{ccc}
M & S & \epsilon N^{T} \\
S^{T} & -\epsilon I & \epsilon J^{T} \\
\epsilon N & \epsilon J & -\epsilon I
\end{array}\right]<0 .
$$

\section{Main results}

Consider the uncertain stochastic system (1) with time varying delays. We take

$$
\begin{aligned}
& y(t)=A(t) x(t)+B(t) x(t-\tau(t)), \\
& g(t)=C(t) x(t)+D(t) x(t-\tau(t)) .
\end{aligned}
$$

Then the system (1) becomes

$$
\mathrm{d} x(t)=y(t) \mathrm{d} t+g(t) \mathrm{d} \omega(t) .
$$

Moreover, the following equality holds:

$$
\begin{aligned}
x(t)-x & (t-\tau(t)) \\
= & \int_{t-\tau(t)}^{t} y(s) \mathrm{d} s+\int_{t-\tau(t)}^{t} g(s) \mathrm{d} \omega(s) .
\end{aligned}
$$

The above equality is used in the proof of the main result. Now the following theorem will discussed without uncertainties.

Theorem 1. For given scalars $\tau_{M}, \tau_{m}$ and $\mu$, the equilibrium point of the stochastic system (1) is asymptotically stable in the mean square sense if there exist matrices $P>0$,

$$
\left[\begin{array}{ll}
Q_{1} & Q_{2} \\
Q_{2}^{T} & Q_{3}
\end{array}\right]>0, \quad\left[\begin{array}{ll}
Q_{4} & Q_{5} \\
Q_{5}^{T} & Q_{6}
\end{array}\right]>0,
$$

$R_{k}>0, k=1 \ldots, 6$, such that for any matrices $N_{i}$ and $M_{i}(i=1, \ldots, 8)$, the following LMI is feasible:

$$
\Omega=\left[\begin{array}{cccc}
\Omega_{11} & \Omega_{12} & \ldots & \Omega_{18} \\
* & \Omega_{22} & \ldots & \Omega_{28} \\
\vdots & \vdots & \ddots & \vdots \\
* & * & \ldots & \Omega_{88}
\end{array}\right]<0
$$


where

$$
\begin{aligned}
& \Omega_{11}=Q_{4}+Q_{1}+R_{1}+R_{2}+R_{3}-R_{4}-R_{5} \\
& +N_{1} A+A^{T} N_{1}^{T}+M_{1} C+C^{T} M_{1}^{T}, \\
& \Omega_{12}=N_{1} B+A^{T} N_{2}^{T}+M_{1} D+C^{T} M_{2}^{T}, \\
& \Omega_{13}=Q_{2}+R_{4}+A^{T} N_{3}^{T}+C^{T} M_{3}^{T}, \\
& \Omega_{14}=A^{T} N_{4}^{T}+C^{T} M_{4}^{T}, \\
& \Omega_{15}=Q_{5}+R_{5}+A^{T} N_{5}^{T}+C^{T} M_{5}^{T}, \\
& \Omega_{16}=A^{T} N_{6}^{T}+C^{T} M_{6}^{T} \text {, } \\
& \Omega_{17}=P+A^{T} N_{7}^{T}+C^{T} M_{7}^{T}-N_{1}, \\
& \Omega_{18}=A^{T} N_{8}^{T}+C^{T} M_{8}^{T}-M_{1} \text {, } \\
& \Omega_{22}=-R_{6}-(1-\mu) R_{1}+N_{2} B+B^{T} N_{2}^{T} \\
& +M_{2} D+D^{T} M_{2}^{T} \text {, } \\
& \Omega_{23}=B^{T} N_{3}^{T}+D^{T} M_{3}^{T}, \\
& \Omega_{24}=R_{6}+B^{T} N_{4}^{T}+D^{T} M_{4}^{T} \text {, } \\
& \Omega_{25}=B^{T} N_{5}^{T}+D^{T} M_{5}^{T} \text {, } \\
& \Omega_{26}=B^{T} N_{6}^{T}+D^{T} M_{6}^{T}, \\
& \Omega_{27}=B^{T} N_{7}^{T}+D^{T} M_{7}^{T}-N_{2} \text {, } \\
& \Omega_{28}=B^{T} N_{8}^{T}+D^{T} M_{8}^{T}-M_{2} \text {, } \\
& \Omega_{33}=Q_{3}-Q_{1}-R_{4}, \quad \Omega_{34}=-Q_{2}, \\
& \Omega_{35}=\Omega_{36}=0, \quad \Omega_{37}=-N_{3} \text {, } \\
& \Omega_{38}=-M_{3}, \quad \Omega_{44}=-Q_{3}-R_{2}-R_{6}, \\
& \Omega_{45}=\Omega_{4,6}=0, \quad \Omega_{47}=-N_{4}, \quad \Omega_{48}=-M_{4} \text {, } \\
& \Omega_{55}=Q_{6}-Q_{4}-R_{5}, \quad \Omega_{56}=-Q_{5}, \\
& \Omega_{57}=-N_{5}, \quad \Omega_{58}=-M_{5} \text {, } \\
& \Omega_{66}=-Q_{6}-R_{3}, \quad \Omega_{67}=-N_{6}, \\
& \Omega_{68}=-M_{6}, \\
& \Omega_{77}=\left(\frac{\tau_{m}}{2}\right)^{2} R_{4}+\left(\frac{\tau_{M}}{2}\right)^{2} R_{5}+\delta^{2} R_{6}-N_{7}-N_{7}^{T}, \\
& \Omega_{78}=-N_{8}^{T}-M_{7}, \\
& \Omega_{88}=P-M_{8}-M_{8}^{T} \text {, } \\
& N=\left[\begin{array}{llllllll}
N_{1}^{T} & N_{2}^{T} & N_{3}^{T} & N_{4}^{T} & N_{5}^{T} & N_{6}^{T} & N_{7}^{T} & N_{8}^{T}
\end{array}\right]^{T},
\end{aligned}
$$

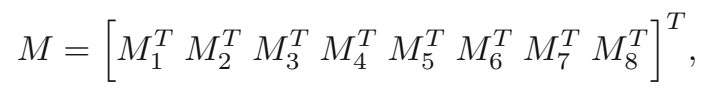

$$
\begin{aligned}
& \delta=\tau_{M}-\tau_{m} .
\end{aligned}
$$

Proof. Consider the Lyapunov-Krasovskii functional

$$
\begin{aligned}
& V\left(x_{t}, t\right) \\
& =V_{1}\left(x_{t}, t\right)+V_{2}\left(x_{t}, t\right)+V_{3}\left(x_{t}, t\right)+V_{4}\left(x_{t}, t\right),
\end{aligned}
$$

\section{where}

$$
\begin{aligned}
& V_{1}\left(x_{t}, t\right)=x^{T}(t) P x(t), \\
& V_{2}\left(x_{t}, t\right) \\
& =\int_{t-\tau(t)}^{t} x^{T}(s) R_{1} x(s) \mathrm{d} s+\int_{t-\tau_{m}}^{t} x^{T}(s) R_{2} x(s) \mathrm{d} s \\
& \quad+\int_{t-\tau_{M}}^{t} x^{T}(s) R_{3} x(s) \mathrm{d} s \\
& V_{3}\left(x_{t}, t\right) \quad \int_{t-\frac{\tau_{m}}{2}}^{t}\left[\begin{array}{c}
x(s) \\
x\left(s-\frac{\tau_{m}}{2}\right)
\end{array}\right]^{T}\left[\begin{array}{ll}
Q_{1} & Q_{2} \\
Q_{2}^{T} & Q_{3}
\end{array}\right]\left[\begin{array}{c}
x(s) \\
x\left(s-\frac{\tau_{m}}{2}\right)
\end{array}\right] \mathrm{d} s \\
& \quad+\int_{t-\frac{\tau_{M}}{2}}^{t}\left[\begin{array}{cc}
x(s) \\
x\left(s-\frac{\tau_{M}}{2}\right)
\end{array}\right]^{T}\left[\begin{array}{ll}
Q_{4} & Q_{5} \\
Q_{5}^{T} & Q_{6}
\end{array}\right]\left[\begin{array}{c}
x(s) \\
x\left(s-\frac{\tau_{M}}{2}\right)
\end{array}\right] \mathrm{d} s,
\end{aligned}
$$

$V_{4}\left(x_{t}, t\right)$

$$
\begin{aligned}
= & \frac{\tau_{m}}{2} \int_{-\frac{\tau_{m}}{2}}^{0} \mathrm{~d} s \int_{t+s}^{t} y^{T}(\theta) R_{4} y(\theta) \mathrm{d} \theta \\
& +\frac{\tau_{M}}{2} \int_{-\frac{\tau_{M}}{2}}^{0} \mathrm{~d} s \int_{t+s}^{t} y^{T}(\theta) R_{5} y(\theta) \mathrm{d} \theta \\
& +\delta \int_{-\tau_{M}}^{-\tau_{m}} \mathrm{~d} s \int_{t+s}^{t} y^{T}(\theta) R_{6} y(\theta) \mathrm{d} \theta .
\end{aligned}
$$

Then it can be obtained by Itō's formula that

$$
\mathrm{d} V\left(x_{t}, t\right)=L V\left(x_{t}, t\right) \mathrm{d} t+2 x^{T}(t) P g(t) \mathrm{d} \omega(t),
$$

where

$$
\begin{aligned}
& L V_{1}\left(x_{t}, t\right)=2 x^{T}(t) P y(t)+g^{T}(t) P g(t), \\
& L V_{2}\left(x_{t}, t\right) \leq x^{T}(t) R_{1} x(t)+x^{T}(t) R_{2} x(t) \\
& -(1-\mu) x^{T}(t-\tau(t)) R_{1} x(t-\tau(t)) \\
& -x^{T}\left(t-\tau_{m}\right) R_{2} x\left(t-\tau_{m}\right)+x^{T}(t) R_{3} x(t) \\
& -x^{T}\left(t-\tau_{M}\right) R_{3} x\left(t-\tau_{M}\right), \\
& L V_{3}\left(x_{t}, t\right)=\left[\begin{array}{c}
x(t) \\
x\left(t-\frac{\tau_{m}}{2}\right)
\end{array}\right]^{T}\left[\begin{array}{ll}
Q_{1} & Q_{2} \\
Q_{2}^{T} & Q_{3}
\end{array}\right]\left[\begin{array}{c}
x(t) \\
x\left(t-\frac{\tau_{m}}{2}\right)
\end{array}\right] \\
& -\left[\begin{array}{c}
x\left(t-\frac{\tau_{m}}{2}\right) \\
x\left(t-\tau_{m}\right)
\end{array}\right]^{T}\left[\begin{array}{ll}
Q_{1} & Q_{2} \\
Q_{2}^{T} & Q_{3}
\end{array}\right]\left[\begin{array}{c}
x\left(t-\frac{\tau_{m}}{2}\right) \\
x\left(t-\tau_{m}\right)
\end{array}\right] \\
& +\left[\begin{array}{c}
x(t) \\
x\left(t-\frac{\tau_{M}}{2}\right)
\end{array}\right]^{T}\left[\begin{array}{ll}
Q_{4} & Q_{5} \\
Q_{5}^{T} & Q_{6}
\end{array}\right]\left[\begin{array}{c}
x(t) \\
x\left(t-\frac{\tau_{M}}{2}\right)
\end{array}\right] \\
& -\left[\begin{array}{c}
x\left(t-\frac{\tau_{M}}{2}\right) \\
x\left(t-\tau_{M}\right)
\end{array}\right]^{T}\left[\begin{array}{ll}
Q_{4} & Q_{5} \\
Q_{5}^{T} & Q_{6}
\end{array}\right]\left[\begin{array}{c}
x\left(t-\frac{\tau_{M}}{2}\right) \\
x\left(t-\tau_{M}\right)
\end{array}\right],
\end{aligned}
$$




$$
\begin{aligned}
& L V_{4}\left(x_{t}, t\right) \\
& =y^{T}(t)\left[\left(\frac{\tau_{m}}{2}\right)^{2} R_{4}+\left(\frac{\tau_{M}}{2}\right)^{2} R_{5}+\delta^{2} R_{6}\right] y(t) \\
& \quad-\frac{\tau_{m}}{2} \int_{t-\frac{\tau_{m}}{2}}^{t} y^{T}(s) R_{4} y(s) \mathrm{d} s \\
& \quad-\frac{\tau_{M}}{2} \int_{t-\frac{\tau_{M}}{2}}^{t} y^{T}(s) R_{5} y(s) \mathrm{d} s \\
& \quad-\delta \int_{t-\tau_{M}}^{t-\tau_{m}} y^{T}(s) R_{6} y(s) \mathrm{d} s, \\
& L V_{4}\left(x_{t}, t\right) \\
& \leq y^{T}(t)\left[\left(\frac{\tau_{m}}{2}\right)^{2} R_{4}+\left(\frac{\tau_{M}}{2}\right)^{2} R_{5}+\delta^{2} R_{6}\right] y(t) \\
& \quad-\frac{\tau_{m}}{2} \int_{t-\frac{\tau_{m}}{2}}^{t} y^{T}(s) R_{4} y(s) \mathrm{d} s \\
& \quad-\frac{\tau_{M}}{2} \int_{t-\frac{\tau_{M}}{2}}^{t} y^{T}(s) R_{5} y(s) \mathrm{d} s \\
& \quad-\delta \int_{t-\tau(t)}^{t-\tau_{m}} y^{T}(s) R_{6} y(s) \mathrm{d} s .
\end{aligned}
$$

Using the note discussed by Kwon et al. (2010), we have

$$
-1=-\left(\tau_{m}\right)^{-1}\left(\frac{\tau_{m}}{2}\right)-\left(1-\left(\tau_{m}\right)^{-1}\left(\frac{\tau_{m}}{2}\right)\right)
$$

Using (11), (15) and Lemma 2, an upper bound of the integral term

$$
-\int_{t-\frac{\tau_{m}}{2}}^{t} y^{T}(s) R_{4} y(s) \mathrm{d} s
$$

can be obtained as

$$
\begin{aligned}
& -\int_{t-\frac{\tau_{m}}{2}}^{t} y^{T}(s) R_{4} y(s) \mathrm{d} s \\
& =-\left(\tau_{m}\right)^{-1}\left(\frac{\tau_{m}}{2}\right) \int_{t-\frac{\tau_{m}}{2}}^{t} y^{T}(s) R_{4} y(s) \mathrm{d} s \\
& -\left[1-\left(\tau_{m}\right)^{-1}\left(\frac{\tau_{m}}{2}\right)\right] \int_{t-\frac{\tau_{m}}{2}}^{t} y^{T}(s) R_{4} y(s) \mathrm{d} s \\
& =-\left(\tau_{m}\right)^{-1}\left(\frac{\tau_{m}}{2}\right) \int_{t-\frac{\tau_{m}}{2}}^{t} y^{T}(s) R_{4} y(s) \mathrm{d} s \\
& \quad-\left(\frac{\tau_{m}}{2}\right)^{-1}\left(1-\left(\tau_{m}\right)^{-1}\left(\frac{\tau_{m}}{2}\right)\right)\left(\frac{\tau_{m}}{2}\right)
\end{aligned}
$$

$$
\begin{aligned}
& \times \int_{t-\frac{\tau_{m}}{2}}^{t} y^{T}(s) R_{4} y(s) \mathrm{d} s \\
\leq & -\left(\tau_{m}\right)^{-1}\left[\int_{t-\frac{\tau_{m}}{2}}^{t} y^{T}(s) \mathrm{d} s\right] R_{4}\left[\int_{t-\frac{\tau_{m}}{2}}^{t} y(s) \mathrm{d} s\right] \\
& -\left(\frac{\tau_{m}}{2}\right)^{-1}\left(1-\left(\tau_{m}\right)^{-1}\left(\frac{\tau_{m}}{2}\right)\right) \\
& \times\left[\int_{t-\frac{\tau_{m}}{2}}^{t} y^{T}(s) \mathrm{d} s\right] R_{4}\left[\int_{t-\frac{\tau_{m}}{2}}^{t} y(s) \mathrm{d} s\right] \\
\leq & -\left(\frac{\tau_{m}}{2}\right)^{-1}\left[\int_{t-\frac{\tau_{m}}{2}}^{t} y^{T}(s) \mathrm{d} s\right] R_{4}\left[\int_{t-\frac{\tau_{m}}{2}}^{t} y(s) \mathrm{d} s\right] \\
= & -\left(\frac{\tau_{m}}{2}\right)^{-1}\left[x(t)-x\left(t-\frac{\tau_{m}}{2}\right)-\int_{t-\frac{\tau_{m}}{2}}^{t} g(s) \mathrm{d} \omega(s)\right] \\
& \left.\times\left[\int_{t-\frac{\tau_{m}}{2}}^{t} g(s) \mathrm{d} \omega(s)\right]^{T} R_{4}\left[x(t)-x\left(t-\frac{\tau_{m}}{2}\right)-\int_{t-\frac{\tau_{m}}{2}}^{t} g(s) \mathrm{d} \omega(s)\right]\right) \\
= & -\left(\frac{\tau_{m}}{2}\right)^{-1}\left(\left[x(t)-x\left(t-\frac{\tau_{m}}{2}\right)\right]^{T}\right. \\
& \times R_{4}\left[x(t)-x\left(t-\frac{\tau_{m}}{2}\right)\right] \\
& -2\left[x(t)-x\left(t-\frac{\tau_{m}}{2}\right)\right]^{T} R_{4}\left[\int_{t}^{t} g(s) \mathrm{d} \omega(s)\right] \\
& \\
& +x(s)]
\end{aligned}
$$

By using

$$
\begin{aligned}
-1= & -\left(\tau_{M}\right)^{-1}\left(\frac{\tau_{M}}{2}\right)-\left(1-\left(\tau_{M}\right)^{-1}\left(\frac{\tau_{M}}{2}\right)\right), \\
-1= & -\left(\tau_{M}-\tau_{m}\right)^{-1}\left(\tau_{M}-\tau_{m}\right) \\
& -\left(1-\left(\tau_{M}-\tau_{m}\right)^{-1}\left(\tau_{M}-\tau_{m}\right)\right)
\end{aligned}
$$

and Lemma 2, upper bounds of the terms

$$
-\int_{t-\frac{\tau_{M}}{2}}^{t} y^{T}(s) R_{5} y(s) \mathrm{d} s
$$

and

$$
-\int_{t-\tau(t)}^{t-\tau_{m}} y^{T}(s) R_{6} y(s) \mathrm{d} s
$$

are respectively obtained as

$$
\begin{aligned}
& -\int_{t-\frac{\tau_{M}}{2}}^{t} y^{T}(s) R_{5} y(s) \mathrm{d} s \\
& \leq-\left(\frac{\tau_{M}}{2}\right)^{-1}\left(\left[x(t)-x\left(t-\frac{\tau_{M}}{2}\right)\right]^{T}\right. \\
& \quad \times R_{5}\left[x(t)-x\left(t-\frac{\tau_{M}}{2}\right)\right]-2\left[x(t)-x\left(t-\frac{\tau_{M}}{2}\right)\right]^{T}
\end{aligned}
$$




$$
\begin{aligned}
& \times R_{5}\left[\int_{t-\frac{\tau_{M}}{2}}^{t} g(s) \mathrm{d} \omega(s)\right]+\left[\int_{t-\frac{\tau_{M}}{2}}^{t} g(s) \mathrm{d} \omega(s)\right]^{T} \\
& \left.R_{5}\left[\int_{t-\frac{\tau_{M}}{2}}^{t} g(s) \mathrm{d} \omega(s)\right]\right) \\
& -\int_{t-\tau(t)}^{t-\tau_{m}} y^{T}(s) R_{6} y(s) \mathrm{d} s \\
& \leq-\left(\tau_{M}-\tau_{m}\right)^{-1}\left(\left[x\left(t-\tau_{m}\right)-x(t-\tau(t))\right]^{T}\right. \\
& \quad \times R_{6}\left[x\left(t-\tau_{m}\right)-x(t-\tau(t))\right] \\
& \quad-2\left[x\left(t-\tau_{m}\right)-x(t-\tau(t))\right]^{T} \\
& \quad \times R_{6}\left[\int_{t-\tau(t)}^{t-\tau_{m}} g(s) \mathrm{d} \omega(s)\right] \\
& \left.\quad+\left[\int_{t-\tau(t)}^{t-\tau_{m}} g(s) \mathrm{d} \omega(s)\right]^{T} R_{6}\left[\int_{t-\tau(t)}^{t-\tau_{m}} g(s) \mathrm{d} \omega(s)\right]\right) .
\end{aligned}
$$

From (8) and (9), for any matrices $N$ and $M$ we have

$$
\begin{aligned}
& 0=2 \xi^{T}(t) N[A(t) x(t)+B(t) x(t-\tau(t))-y(t)], \\
& 0=2 \xi^{T}(t) M[C(t) x(t)+D(t) x(t-\tau(t))-g(t)] .
\end{aligned}
$$

Substituting (16)-(18) into (14) and adding (19)-(20), we have

$$
L V\left(x_{t}, t\right) \leq \xi^{T}(t) \Omega \xi(t)+2(\zeta(t) \mathrm{d} \omega(t)),
$$

where

$$
\begin{aligned}
\xi^{T}(t)= & {\left[x^{T}(t) x^{T}(t-\tau(t)) x^{T}\left(t-\frac{\tau_{m}}{2}\right) x^{T}\left(t-\tau_{m}\right)\right.} \\
& \left.\times x^{T}\left(t-\frac{\tau_{M}}{2}\right) x^{T}\left(t-\tau_{M}\right) y^{T}(t) g^{T}(t)\right] .
\end{aligned}
$$

$(\zeta(t) \mathrm{d} \omega(t))$

$$
\begin{aligned}
& =\frac{1}{\tau_{m}}\left[x(t)-x\left(t-\frac{\tau_{m}}{2}\right]^{T} R_{4}\left[\int_{t-\frac{\tau_{m}}{2}}^{t} g(s) \mathrm{d} \omega(s)\right]\right. \\
& +\frac{1}{\tau_{M}}\left[x(t)-x\left(t-\frac{\tau_{M}}{2}\right]^{T} R_{5}\left[\int_{t-\frac{\tau_{M}}{2}}^{t} g(s) \mathrm{d} \omega(s)\right]\right. \\
& +\frac{1}{\tau_{M}-\tau_{m}}\left[x\left(t-\tau_{m}\right)-x(t-\tau(t))\right]^{T} \\
& \times R_{6}\left[\int_{t-\tau(t)}^{t-\tau_{m}} g(s) \mathrm{d} \omega(s)\right]+x^{T} P g(t) \mathrm{d} \omega(t) .
\end{aligned}
$$

Taking expectation on both sides of (21), we have

$$
\mathbb{E}\left\{L V\left(x_{t}, t\right)\right\} \leq \mathbb{E}\left\{\xi^{T}(t) \Omega \xi(t)\right\} .
$$

Now we proceed to prove that the system (1) is stochastically stable by using a similar method as Chen et al. (2004). Set $\lambda_{0}=\lambda_{\min }(-\Omega)$, then $\lambda_{0}>0$ follows from (12). From (22) and by using Itō's formula (Shi and Boukas, 1997),

$$
\begin{aligned}
\mathbb{E} V(t)-\mathbb{E} V\left(\tau_{M}\right) & =\mathbb{E} \int_{\tau_{M}}^{t} L V(s) \mathrm{d} s \\
& \leq-\lambda_{0} \mathbb{E} \int_{\tau_{M}}^{t}\|x(s)\|^{2} \mathrm{~d} s .
\end{aligned}
$$

It follows that

$$
\mathbb{E} \int_{\tau_{M}}^{t}\|x(s)\|^{2} \mathrm{~d} s \leq \frac{1}{\lambda_{0}} \mathbb{E} V\left(\tau_{M}\right) .
$$

For the system (1), following Chen et al. (2004) it is easy to prove that there exists a positive scalar $c_{1} \geq 1$ such that

$$
\mathbb{E}\|x(s)\|^{2} \leq c_{1} \sup _{s \in\left[-\tau_{M}, 0\right]} \mathbb{E}\|\phi(s)\|^{2}, \quad t \in\left[0, \tau_{M}\right] .
$$

Therefore, by the definitions of $V(t)$ and $x(t)$, there always exists a scalar $c>0$ such that

$$
\lim _{T \longrightarrow \infty} \mathbb{E} \int_{0}^{T}\|x(s)\|^{2} \mathrm{~d} s \leq c \sup _{s \in\left[-\tau_{M}, 0\right]} \mathbb{E}\|\phi(s)\|^{2},
$$

which means that the system (1) is stochastically stable by Definition 1. This completes the proof.

Remark 1. Theorem 1 provides delay-dependent stability criteria for the stochastic system (1). Such criteria are derived based on the assumption that the time-varying delay is differentiable and the value of $\mu$ is known. The conditions in Theorem 1 are formulated in terms of the solvability of LMIs (Boyd et al., 1994) and can be easily solved using MATLAB LMI Control Toolbox. It is worth noting that, by applying convex optimization algorithms, we can conclude that the maximum allowable upper bound of the interval time-varying delay, that is, $\tau_{M}$, guarantees the feasibility of the presented LMIs.

We can obtain the maximum allowable upper bound $\tau_{M}$ by solving the following optimization problem:

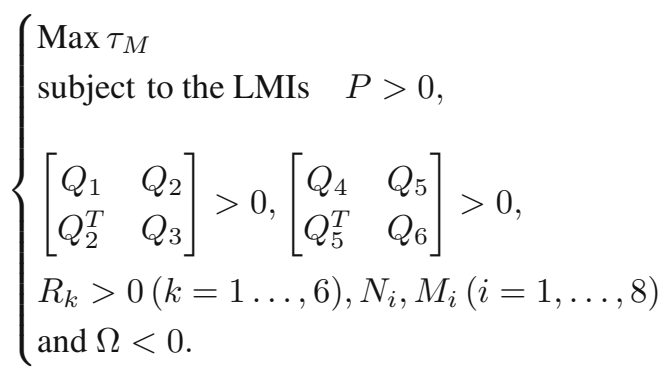

Remark 2. In this paper, in order to derive the stability criterion, we employ a new Lyapunov-Krasovskii functional (13), which is mainly based on the information about $\tau_{m} / 2, \tau_{M} / 2$ and $\left(\tau_{M}-\tau_{m}\right)$; some suitable freeweight matrices are also introduced. 
Theorem 2. For given scalars $\tau_{M}, \tau_{m}$ and $\mu$, the equilibrium point of the stochastic system (1) subject to the polytopic uncertainty (3) is asymptotically stable in the mean square if there exist matrices $P>0$,

$$
\begin{aligned}
& {\left[\begin{array}{cc}
Q_{1}^{(i)} & Q_{2}^{(i)} \\
Q_{2}^{(i) T} & Q_{3}^{(i)}
\end{array}\right]>0,} \\
& {\left[\begin{array}{cc}
Q_{4}^{(i)} & Q_{5}^{(i)} \\
Q_{5}^{(i) T} & Q_{6}^{(i)}
\end{array}\right]>0,}
\end{aligned}
$$

$(i=1,2, \ldots, r), R_{k}>0, k=1 \ldots, 6$ such that for any $m a-$ trices $N_{i}$ and $M_{i},(i=1, \ldots, 8)$ the following LMI is feasible:

$$
\bar{\Omega}_{i}<0,
$$

for $i=1,2, \ldots, r$, where

$$
\bar{\Omega}_{i}=\left[\begin{array}{cccc}
\bar{\Omega}_{11, i} & \bar{\Omega}_{12, i} & \ldots & \bar{\Omega}_{18, i} \\
* & \bar{\Omega}_{22, i} & \ldots & \bar{\Omega}_{28, i} \\
\vdots & \vdots & \ddots & \vdots \\
* & * & \ldots & \bar{\Omega}_{88, i}
\end{array}\right]<0,
$$

with

$$
\begin{aligned}
\bar{\Omega}_{11, i}= & Q_{4}^{(i)}+Q_{1}^{(i)}+R_{1}+R_{2}+R_{3}-R_{4}-R_{5} \\
& +N_{1} A^{(i)}+A^{(i) T} N_{1}^{T}+M_{1} C^{(i)}+C^{(i) T} M_{1}^{T}, \\
\bar{\Omega}_{12, i}= & N_{1} B^{(i)}+A^{(i) T} N_{2}^{T}+M_{1} D^{(i)}+C^{(i) T} M_{2}^{T}, \\
\bar{\Omega}_{13, i}= & Q_{2}^{(i)}+R_{4}+A^{(i) T} N_{3}^{T}+C^{(i) T} M_{3}^{T}, \\
\bar{\Omega}_{14, i}= & A^{(i) T} N_{4}^{T}+C^{(i) T} M_{4}^{T}, \\
\bar{\Omega}_{15, i}= & Q_{5}^{(i)}+R_{5}+A^{(i) T} N_{5}^{T}+C^{(i) T} M_{5}^{T}, \\
\bar{\Omega}_{16, i}= & A^{(i) T} N_{6}^{T}+C^{(i) T} M_{6}^{T}, \\
\bar{\Omega}_{17, i}= & P+A^{(i) T} N_{7}^{T}+C^{(i) T} M_{7}^{T}-N_{1}, \\
\bar{\Omega}_{18, i}= & A^{(i) T} N_{8}^{T}+C^{(i) T} M_{8}^{T}-M_{1}, \\
\bar{\Omega}_{22, i}= & -R_{6}-(1-\mu) R_{1}+N_{2} B^{(i)}+B^{(i) T} N_{2}^{T} \\
& +M_{2} D^{(i)}+D^{(i) T} M_{2}^{T}, \\
\bar{\Omega}_{28, i}= & B^{(i) T} N_{8}^{T}+D^{(i) T} M_{8}^{T}-M_{2}, \\
\bar{\Omega}_{23, i}= & B^{(i) T} N_{3}^{T}+D^{(i) T} M_{3}^{T}, \\
\bar{\Omega}_{24, i}= & R_{6}+B^{(i) T} N_{4}^{T}+D^{(i) T} M_{4}^{T}, \\
\bar{\Omega}_{25, i}= & B^{(i) T} N_{5}^{T}+D^{(i) T} M_{5}^{T}, \\
\bar{\Omega}_{26, i}= & B_{6}^{(i) T} N_{7}^{T}+D^{(i) T} M_{6}^{T}, \\
& \\
& \\
&
\end{aligned}
$$

$$
\begin{aligned}
& \bar{\Omega}_{33, i}=Q_{3}^{(i)}-Q_{1}^{(i)}-R_{4}, \quad \bar{\Omega}_{34, i}=-Q_{2}^{(i)}, \\
& \bar{\Omega}_{35, i}=\Omega_{36, i}=0, \quad \bar{\Omega}_{37, i}=-N_{3}, \quad \bar{\Omega}_{38, i}=-M_{3}, \\
& \bar{\Omega}_{44, i}=-Q_{3}^{(i)}-R_{2}-R_{6}, \quad \bar{\Omega}_{45, i}=\bar{\Omega}_{4,6, i}=0, \\
& \bar{\Omega}_{47, i}=-N_{4}, \quad \bar{\Omega}_{48, i}=-M_{4}, \\
& \bar{\Omega}_{55, i}=Q_{6}^{(i)}-Q_{4}^{(i)}-R_{5}, \\
& \bar{\Omega}_{56, i}=-Q_{5}^{(i)}, \quad \bar{\Omega}_{57, i}=-N_{5}, \quad \bar{\Omega}_{58, i}=-M_{5}, \\
& \bar{\Omega}_{66, i}=-Q_{6}^{(i)}-R_{3}, \bar{\Omega}_{67, i}=-N_{6}, \quad \bar{\Omega}_{68, i}=-M_{6}, \\
& \bar{\Omega}_{77, i}=\left(\frac{\tau_{m}}{2}\right)^{2} R_{4}+\left(\frac{\tau_{M}}{2}\right)^{2} R_{5}+\delta^{2} R_{6}-N_{7}-N_{7}^{T}, \\
& \bar{\Omega}_{78, i}=-N_{8}^{T}-M_{7}, \quad \bar{\Omega}_{88, i}=P-M_{8}-M_{8}^{T},
\end{aligned}
$$

Proof. By Schur's complement, the matrix inequality (23) implies

$$
\sum_{i=1}^{r} \lambda_{i} \bar{\Omega}_{i}<0
$$

or $\Omega<0$, where

$$
\begin{aligned}
A & =\sum_{i=1}^{r} \lambda_{i} A^{(i)}, & B & =\sum_{i=1}^{r} \lambda_{i} B^{(i)}, \\
C & =\sum_{i=1}^{r} \lambda_{i} C^{(i)}, & D & =\sum_{i=1}^{r} \lambda_{i} D^{(i)}, \\
Q & =\sum_{i=1}^{r} \lambda_{i} Q_{j}^{(i)}, & &
\end{aligned}
$$

$j=1,2, \ldots, 6$. This completes the proof.

Similarly to the proof of Theorem 1, we can establish the following result.

Theorem 3. For given scalars $\tau_{M}, \tau_{m}$ and $\mu$, the equilibrium point of the stochastic system (1) subject to the linear fractional norm-bounded uncertainty (4) is robustly asymptotically stable in the mean square if there exist scalars $\epsilon_{1}>0, \epsilon_{2}>0$, matrices $P>0$,

$$
\left[\begin{array}{ll}
Q_{1} & Q_{2} \\
Q_{2}^{T} & Q_{3}
\end{array}\right]>0, \quad\left[\begin{array}{ll}
Q_{4} & Q_{5} \\
Q_{5}^{T} & Q_{6}
\end{array}\right]>0,
$$

$R_{k}>0, k=1 \ldots, 6$, such that for any matrices $N_{i}$ and $M_{i}(i=1, \ldots, 8)$, the following LMI is feasible:

$$
\left[\begin{array}{ccccc}
\Omega & N H & \epsilon_{1} S_{1}^{T} & M H & \epsilon_{2} S_{2}^{T} \\
* & -\epsilon_{1} I & \epsilon_{1} J^{T} & 0 & 0 \\
* & * & -\epsilon_{1} I & 0 & 0 \\
* & * & * & -\epsilon_{2} I & \epsilon_{2} J^{T} \\
* & * & * & * & -\epsilon_{2} I
\end{array}\right]<0,
$$

$\Omega$ being defined in (12). 
Proof. Assume that the inequality (24) holds. It can be seen that (24) can be rewritten as

$$
\begin{aligned}
\Psi & =\left[\begin{array}{ccccc}
\Omega & N H & \epsilon_{1} S_{1}^{T} & M H & \epsilon_{2} S_{2}^{T} \\
* & -\epsilon_{1} I & \epsilon_{1} J^{T} & 0 & 0 \\
* & * & -\epsilon_{1} I & 0 & 0 \\
* & * & * & -\epsilon_{2} I & \epsilon_{2} J^{T} \\
* & * & * & * & -\epsilon_{2} I
\end{array}\right]<0, \\
S_{1} & =\left[\begin{array}{llllllll}
E & E_{1} & 0 & 0 & 0 & 0 & 0 & 0
\end{array}\right]^{T}, \\
S_{2} & =\left[\begin{array}{llllllll}
E_{2} & E_{3} & 0 & 0 & 0 & 0 & 0 & 0
\end{array}\right]^{T} .
\end{aligned}
$$

Thus,

$$
\begin{aligned}
\Psi= & \Omega+N \Lambda(t) S_{1}+S_{1}^{T} \Lambda(t) N^{T} \\
& +M \Lambda(t) S_{2}+S_{2}^{T} \Lambda(t) M^{T}<0
\end{aligned}
$$

holds according to Lemma 3. It can be verified that $\Psi$ is exactly the same as $\Omega$ of (12) when $A, B, C$ and $D$ are replaced respectively by $A+H \Lambda(t) E, B+H \Lambda(t) E_{1}$, $C+H \Lambda(t) E_{2}$ and $D+H \Lambda(t) E_{3}$ in (12).

Now, without considering the terms $\tau_{M} / 2$ and $\tau_{m} / 2$ in the Lyapunov-Krasovskii functional, the corresponding result is discussed in the following corollary.

Corollary 1. For given scalars $\tau_{M}, \tau_{m}$ and $\mu$, the equilibrium point of the stochastic system (1) subject to the linear fractional norm-bounded uncertainty (4) is robustly asymptotically stable in the mean square if there exist scalars $\epsilon_{1}>0, \epsilon_{2}>0$, matrices $P>0, R_{1}>0$, $k=1,2,3, R_{6}>0$ for any matrices $\bar{N}_{i}$ and $\bar{M}_{i}(i=$ $1, \ldots, 6)$ the following LMI is feasible:

$$
\left[\begin{array}{ccccc}
\Xi & \bar{N} H & \epsilon_{1} \bar{S}_{1}^{T} & \bar{M} H & \epsilon_{2} \bar{S}_{2}^{T} \\
* & -\epsilon_{1} I & \epsilon_{1} J^{T} & 0 & 0 \\
* & * & -\epsilon_{1} I & 0 & 0 \\
* & * & * & -\epsilon_{2} I & \epsilon_{2} J^{T} \\
* & * & * & * & -\epsilon_{2} I
\end{array}\right]<0
$$

where $\Xi=\left(\Xi_{n \times m}\right)_{6 \times 6}$ with

$$
\begin{aligned}
\Xi_{11}= & R_{1}+R_{2}+R_{3}+N_{1} A+A^{T} N_{1}^{T} \\
& +M_{1} C+C^{T} M_{1}^{T}, \\
\Xi_{12}= & N_{1} B+A^{T} N_{2}^{T}+M_{1} D+C^{T} M_{2}^{T}, \\
\Xi_{13}= & A^{T} N_{3}^{T}+C^{T} M_{3}^{T}, \\
\Xi_{14}= & A^{T} N_{4}^{T}+C^{T} M_{4}^{T}, \\
\Xi_{15}= & P+A^{T} N_{5}^{T}+C^{T} M_{5}^{T}-N_{1},
\end{aligned}
$$

$$
\begin{aligned}
& \Xi_{16}=A^{T} N_{6}^{T}+C^{T} M_{6}^{T}-M_{1}, \\
& \Xi_{22}=-(1-\mu) R_{1}-R_{6}+N_{2} B+B^{T} N_{2}^{T} \\
& +M_{2} D+D^{T} M_{2}^{T} \text {, } \\
& \Xi_{23}=B^{T} N_{3}^{T}+D^{T} M_{3}^{T}+R_{6}, \\
& \Xi_{24}=B^{T} N_{4}^{T}+D^{T} M_{4}^{T}, \\
& \Xi_{25}=B^{T} N_{5}^{T}+D^{T} M_{5}^{T}-N_{2}, \\
& \Xi_{26}=B^{T} N_{6}^{T}+D^{T} M_{6}^{T}-M_{2}, \\
& \Xi_{33}=-R_{2}-R_{6}, \quad \Omega_{34}=0, \quad \Xi_{35}=-N_{3}, \\
& \Xi_{36}=-M_{3}, \\
& \Omega_{44}=-R_{3}, \Xi_{45}-N_{4}, \quad \Xi_{46}=-M_{4},, \\
& \Xi_{55}=\delta^{2} R_{6}-N_{5}-N_{5}^{T}, \quad \Xi_{56}=-N_{6}^{T}-M_{5}, \\
& \Omega_{66}=P-M_{6}-M_{6}^{T}, \\
& \bar{N}=\left[\begin{array}{llllll}
N_{1}^{T} & N_{2}^{T} & N_{3}^{T} & N_{4}^{T} & N_{5}^{T} & N_{6}^{T}
\end{array}\right]^{T}, \\
& \bar{M}=\left[\begin{array}{lllll}
M_{1}^{T} & M_{2}^{T} & M_{3}^{T} & M_{4}^{T} & M_{5}^{T} \\
M_{6}^{T}
\end{array}\right]^{T}, \\
& \bar{S}_{1}=\left[\begin{array}{llllll}
E & E_{1} & 0 & 0 & 0 & 0
\end{array}\right]^{T}, \\
& \bar{S}_{2}=\left[\begin{array}{llllll}
E_{2} & E_{3} & 0 & 0 & 0 & 0
\end{array}\right]^{T} \text {. }
\end{aligned}
$$

Proof. Consider the Lyapunov-Krasovskii functional

$$
V\left(x_{t}, t\right)=V_{1}\left(x_{t}, t\right)+V_{2}\left(x_{t}, t\right)+V_{3}\left(x_{t}, t\right),
$$

where

$$
\begin{aligned}
& V_{1}\left(x_{t}, t\right)= x^{T}(t) P x(t), \\
& V_{2}\left(x_{t}, t\right)= \int_{t-\tau(t)}^{t} x^{T}(s) R_{1} x(s) \mathrm{d} s \\
&+\int_{t-\tau_{m}}^{t} x^{T}(s) R_{2} x(s) \mathrm{d} s \\
&+\int_{t-\tau_{M}}^{t} x^{T}(s) R_{3} x(s) \mathrm{d} s, \\
& V_{3}\left(x_{t}, t\right)=\delta \int_{-\tau_{M}}^{-\tau_{m}} \mathrm{~d} s \int_{t+s}^{t} y^{T}(\theta) R_{6} y(\theta) \mathrm{d} \theta .
\end{aligned}
$$

Then, it can be obtained by Itō's formula that

$$
\mathrm{d} V\left(x_{t}, t\right)=L V\left(x_{t}, t\right) \mathrm{d} t+2 x^{T}(t) P g(t) \mathrm{d} \omega(t),
$$


where

$$
\begin{aligned}
& \quad L V_{1}\left(x_{t}, t\right) \\
& =2 x^{T}(t) P y(t)+g^{T}(t) P g(t), \\
& L V_{2}\left(x_{t}, t\right) \\
& \leq x^{T}(t) R_{1} x(t) \\
& \quad-(1-\mu) x^{T}(t-\tau(t)) R_{1} x(t-\tau(t)) x^{T}(t) R_{2} x(t) \\
& \quad-x^{T}\left(t-\tau_{m}\right) R_{2} x\left(t-\tau_{m}\right)+x^{T}(t) R_{3} x(t) \\
& \quad-x^{T}\left(t-\tau_{M}\right) R_{3} x\left(t-\tau_{M}\right), \\
& L V_{3}\left(x_{t}, t\right) \\
& =y^{T}(t) \delta^{2} R_{6} y(t) \\
& \quad-\delta \int_{t-\tau_{M}}^{t-\tau_{m}} y^{T}(s) R_{6} y(s) \mathrm{d} s \\
& L V_{3}\left(x_{t}, t\right) \\
& \leq y^{T}(t) \delta^{2} R_{6} y(t) \\
& -\delta \int_{t-\tau(t)}^{t-\tau_{m}} y^{T}(s) R_{6} y(s) \mathrm{d} s .
\end{aligned}
$$

From (8) and (9) for any matrices $N$ and $M$ we have

$$
\begin{aligned}
& 0=2 \xi_{1}^{T}(t) N[A(t) x(t)+B(t) x(t-\tau(t))-y(t)], \\
& 0=2 \xi_{1}^{T}(t) M[C(t) x(t)+D(t) x(t-\tau(t))-g(t)] .
\end{aligned}
$$

where

$$
\begin{aligned}
\xi_{1}^{T}(t)= & {\left[x^{T}(t) x^{T}(t-\tau(t)) x^{T}\left(t-\tau_{m}\right)\right.} \\
& \left.x^{T}\left(t-\tau_{M}\right) y^{T}(t) g^{T}(t)\right] .
\end{aligned}
$$

Following similar arguments as in the proof of Theorem 3, we can obtain the desired result immediately, and hence the detailed proof is omitted.

Remark 3. It is easy to see that, while setting $J=0$, the linear fractional norm-bounded uncertainty reduces to a routine norm-bounded uncertainty. Therefore, one can easily derive a corresponding result for the routine normbounded uncertainty from Theorem 3 .

\section{Numerical examples}

In this section, we will give four examples to show the effectiveness of the established theoretical results.
4.1. Example. Consider the system (1) and (3) with the following matrices:

$$
\begin{array}{rlrl}
A^{1} & =\left[\begin{array}{cc}
-2 & 0 \\
0 & -1
\end{array}\right], & A^{2} & =\left[\begin{array}{cc}
-2 & -1 \\
0 & -2
\end{array}\right], \\
B^{1} & =\left[\begin{array}{cc}
-0.1 & 0 \\
-0.1 & -0.1
\end{array}\right], & B^{2}=\left[\begin{array}{ll}
0 & 1 \\
1 & 0
\end{array}\right], \\
C^{1}=\left[\begin{array}{cc}
-0.2 & 0 \\
0 & -0.2
\end{array}\right], & C^{2}=\left[\begin{array}{cc}
0.2 & 0 \\
0 & 0.1
\end{array}\right], \\
D^{1}=\left[\begin{array}{cc}
0.5 & 0 \\
0 & 0.7
\end{array}\right], & D^{2}=\left[\begin{array}{cc}
0.2 & 0 \\
0 & -0.1
\end{array}\right] .
\end{array}
$$

It was reported by Li et al. (2008) that the above system is robustly asymptotically stable in the mean square when the constant delay $\tau_{M}=5$. However, by Theorem 2 and using Matlab LMI Toolbox for $\mu=0, \tau_{m}=0$, it is found that the equilibrium point of an uncertain stochastic system is asymptotically stable in the mean square for any constant allowable upper bounds. This implies that our stability criterion gives a less conservative result than the methods discussed by Li et al. (2008).

4.2. Example. Consider the system (1) and (3) with the following matrices:

$$
\begin{array}{rlrl}
A^{1} & =\left[\begin{array}{cc}
-2 & 0 \\
0 & -1
\end{array}\right], & A^{2} & =\left[\begin{array}{cc}
-1.5 & -1 \\
0 & -2
\end{array}\right], \\
B^{1} & =\left[\begin{array}{cc}
1 & 0 \\
-0.1 & 0.85
\end{array}\right], & B^{2} & =\left[\begin{array}{cc}
-1 & 1 \\
1 & 0.85
\end{array}\right], \\
C^{1} & =\left[\begin{array}{cc}
-0.2 & 0 \\
0 & -0.2
\end{array}\right], & C^{2} & =\left[\begin{array}{cc}
0.2 & 0 \\
0 & 0.1
\end{array}\right], \\
D^{1} & =\left[\begin{array}{cc}
0.5 & 0 \\
0 & 0.7
\end{array}\right], & D^{2}=\left[\begin{array}{cc}
0.2 & 0 \\
0 & -0.1
\end{array}\right] .
\end{array}
$$

It was reported by Li et al. (2008) that the above system is robustly asymptotically stable in the mean square for a constant delay of $\tau_{M}=2.4019$. However, by our Theorem 2 and using Matlab LMI Toolbox for $\mu=0$, $\tau_{m}=0$, it is found that the equilibrium point of an uncertain stochastic system is asymptotically stable in the mean square for any constant allowable upper bounds. This implies that our stability criterion gives a less conservative result than the methods discussed by Li et al. (2008).

4.3. Example. Consider the uncertain stochastic system with time-varying delay described by

$$
\begin{aligned}
& \mathrm{d} x(t) \\
& =[(A+\Delta A(t)) x(t)+(B+\Delta B(t)) x(t-\tau(t))] \mathrm{d} t \\
& \quad+[\Delta C(t) x(t)+\Delta D(t) x(t-\tau(t))] \mathrm{d} w(t),
\end{aligned}
$$


where

$$
\begin{array}{cl}
A=\left[\begin{array}{cc}
-2 & 0 \\
0 & -0.9
\end{array}\right], & B=\left[\begin{array}{cc}
-1 & 0 \\
-1 & -1
\end{array}\right], \\
\|\Delta A(t)\| \leq 0.2, & \\
\|\Delta B(t)\| \leq 0.2, \\
\|\Delta C(t)\| \leq 0.2, & \|\Delta D(t)\| \leq 0.2 .
\end{array}
$$

Then take the uncertainties as described by (4) as follows

$$
\begin{aligned}
& H=\left[\begin{array}{cc}
0.2 & 0 \\
0 & 0.2
\end{array}\right], \\
& E=E_{1}=E_{2}=E_{3}=\left[\begin{array}{ll}
1 & 0 \\
0 & 1
\end{array}\right] .
\end{aligned}
$$

According to Theorem 3, the upper bounds on the time delay to guarantee that the system is robustly stochastically stable are listed in Tables 1 and 2. Table 1 also lists the upper bounds obtained from the criterion by Miyamura and Aihara (2004), Yan et al. (2009), Zhang et al. (2009) and Zhang et al. (2008). Hence the method proposed in this paper gives less conservative results than the existing results found in the literature (Miyamura and Aihara, 2004; Yan et al., 2009; Zhang et al., 2009; Zhang et al., 2008).

4.4. Example. Consider the uncertain stochastic system with time-varying delay described by

$$
\begin{aligned}
& \mathrm{d} x(t) \\
& =[(A+\Delta A(t)) x(t)+(B+\Delta B(t)) x(t-\tau(t))] \mathrm{d} t \\
& \quad+[C x(t)+D x(t-\tau(t))] \mathrm{d} w(t),
\end{aligned}
$$

where

$$
\begin{aligned}
& A=\left[\begin{array}{cc}
-2 & 0 \\
1 & -1
\end{array}\right], \quad B=\left[\begin{array}{cc}
-1 & 0 \\
-0.5 & -1
\end{array}\right], \\
& \|\Delta A(t)\| \leq 0.1, \quad\|\Delta B(t)\| \leq 0.1 \\
& C=D=\operatorname{diag}(\sqrt{0.1}, \sqrt{0.1}) \\
& E=E_{1}=\operatorname{diag}(0.1,0.1) \\
& H=\left[\begin{array}{cc}
1 & 0 \\
0 & 1
\end{array}\right] .
\end{aligned}
$$

According to Theorem 3, the upper bounds on the time delay to guarantee that the system is robustly stochastically stable are listed in Table 3, which also gives the upper bounds obtained from the criterion by Yue and Han (2005) as well as He et al. (2010). Hence the method proposed in this paper gives less conservative results than the results found in the literature (Yue and Han, 2005; He et al., 2010).

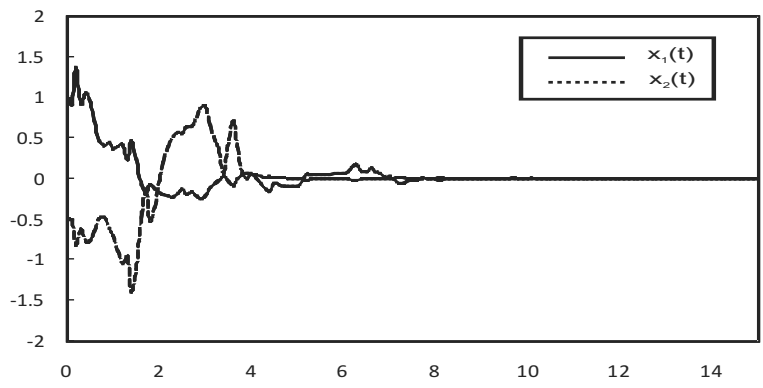

Fig. 1. State trajectories of $x_{1}$ and $x_{2}$ for Example 4.3 with different initial conditions $(1,-0.5)$.

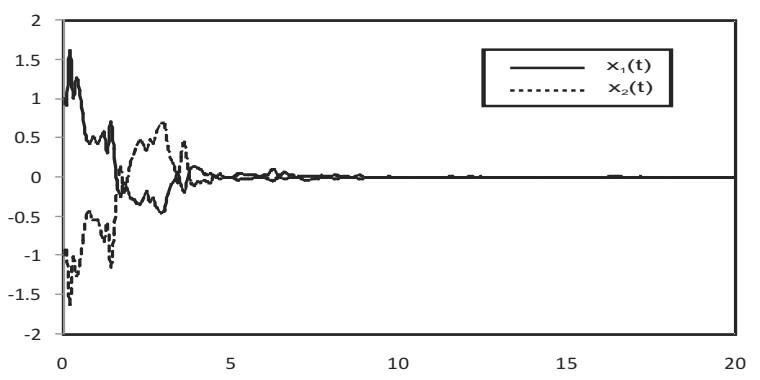

Fig. 2. State trajectories of $x_{1}$ and $x_{2}$ for Example 4.4 with different initial conditions $(1,-1)$.

\section{Conclusion}

Several sufficient conditions ensuring an LMI optimization problem of delay-dependent robust stability criteria for stochastic systems with polytopic and linear fractional uncertainties have been proposed. By choosing a suitable Lyapunov-Krasovskii functional and the free-weighting matrix method, some less conservative stability criteria have been obtained. The restriction that the derivative of the time-varying delay is less than one has been removed. In numerical comparisons, significant improvements over the recent existing results have been observed.

\section{Acknowledgment}

The authors are very much thankful to the referees for their valuable comments and suggestions for improving this manuscript.

\section{References}

Balasubramaniam, P., Lakshmanan, S. and Rakkiyappan, R. (2009). Delay-interval dependent robust stability criteria for stochastic neural networks with linear fractional uncertainties, Neurocomputing 72(16-18): 3675-3682.

Balasubramaniam, P. and Lakshmanan, S. (2011). Delay-interval dependent robust stability criteria for neutral stochastic neural networks with polytopic and linear fractional uncer- 
Table 1. Maximum allowable upper bound of $h_{2}$ with different $\mu, h_{1}=0$ and $J=0$.

\begin{tabular}{|c|c|c|c|c|c|}
\hline$\mu$ & 0 & 0.3 & 0.5 & 0.9 & 1 \\
\hline \hline Miyamura and Aihara, 2004 & 0.3555 & - & - & - & - \\
\hline Yan et al., 2009 & 1.0660 & 0.7288 & 0.5252 & 0.1489 & - \\
\hline Zhang et al., 2009 & - & 1.2950 & 1.1006 & 0.9434 & 0.9424 \\
\hline Zhang et al., 2008 & 1.8684 & - & 1.1304 & 0.9402 & - \\
\hline Theorem 3 & 2.4670 & 1.6880 & 1.3428 & 0.9656 & 0.9428 \\
\hline
\end{tabular}

Table 2. Maximum allowable upper bound of $h_{2}$ with different $\mu, h_{1}$ and $J$.

\begin{tabular}{|c|c|c|c|c|c|}
\hline$\mu$ & 0 & 0.3 & 0.5 & 1 & 2 \\
\hline \hline$J=0, h_{1}=0.5$ & 2.1952 & 1.5607 & 1.2709 & 0.9359 & 0.9359 \\
\hline$J=0, h_{1}=1$ & 2.1929 & 1.6065 & 1.3593 & 1.3149 & 1.3149 \\
\hline$J=0.5, h_{1}=0.5$ & 1.6603 & 1.2994 & 1.0947 & 0.8828 & 0.8828 \\
\hline
\end{tabular}

Table 3. Maximum allowable upper bound of $h_{2}$. $\left(J=0, h_{1}=0\right),\left(J=0, h_{1}=0.5\right),\left(J=0, h_{1}=1\right),\left(J=0.5, h_{1}=0.5\right)$ are denoted by $a *, b *, c *, d *$, respectively.

\begin{tabular}{|c|c|c|c|c|c|c|}
\hline$\mu$ & 0 & 0.3 & 0.5 & 0.9 & 1 & 2 \\
\hline \hline Yue and Han, 2005 & - & - & 0.8502 & 0.4606 & 0.5001 & - \\
\hline He et al., 2010 & - & - & 0.9010 & 0.7312 & 0.7312 & - \\
\hline$a *$ & 8.9548 & 2.4868 & 1.7940 & 0.9849 & 0.8264 & 0.8264 \\
\hline$b *$ & 8.9585 & 2.4955 & 1.8055 & 1.1016 & 1.1016 & 1.1016 \\
\hline$c *$ & 8.9725 & 2.5354 & 1.8602 & 1.4679 & 1.4679 & 1.4679 \\
\hline$d *$ & 6.6322 & 2.4472 & 1.8017 & 1.1188 & 1.1188 & 1.1188 \\
\hline
\end{tabular}

tainties, International Journal of Computer Mathematics 88(10): 2001-2015.

Boyd, S., El Ghaouli, L., Feron, E. and Balakrishnan, V. (1994). Linear Matrix Inequalities in System and Control Theory, SIAM, Philadelphia, PA.

Chen, W.Y. (2002). Some new results on the asymptotic stability of uncertain systems with time-varying delays, International Journal of Systems Science 33(11): 917-21.

Chen, W.H., Guan, Z.H. and Lu, X.M. (2004). Delay-dependent robust stabilization and $H_{\infty}$ control of uncertain stochastic systems with time-varying delay, IMA Journal of Mathematical Control Information 21(3): 345-58.

Chen, W.H., Guan, Z.H. and Lu, X.M. (2005). Delay-dependent exponential stability of uncertain stochastic systems with multiple delays: An LMI approach, Systems \& Control Letters 54(6): 547-555.

Chesi, G., Garulli, A., Tesi, A. and Vicino, A. (2007). Robust stability of time-varying polytopic systems via parameterdependent homogeneous Lyapunov functions, Automatica 43(2): 309-316.

Geromel, J.C. and Colaneri, P. (2006). Robust stability of time varying polytopic systems, Systems \& Control Letters 55(1): 81-85.

Gu, K., Kharitonov, V.L. and Chen, J. (2003). Stability of Timedelay Systems, Birkhauser, Boston, MA.

$\mathrm{Gu}$, K. (2000). An integral inequality in the stability problem of time-delay systems, Proceedings of the 39th IEEE Conference on Decision and Control, Sydney, Australia, pp. 2805-2810.
Hale, J.K. and Verduyn Lunel, S.M. (1993). Introduction to Functional Differential Equations, Springer, New York, NY.

He, Y., Wang, Q.-G. and Lin, C. (2006). An improved $H_{\infty}$ filter design for systems with time-varying interval delay, IEEE Transactions on Circuits and Systems II: Express Briefs 53(11): 1235-1239.

He, Y., Wu, M., She, J.H. and Liu, G.P. (2004). Parameterdependent Lyapunov functional for stability of time-delay systems with polytopic-type uncertainties, IEEE Transactions on Automatic Control 49(5): 828-832.

He, Y., Zhang, Y., Wu, M. and She, J.-H. (2010). Improved exponential stability for stochastic Markovian jump systems with nonlinearity and time-varying delay, International Journal of Robust and Nonlinear Control 20(1): 1626.

Huang, Y. and Zhou, K. (2000). Robust stability of uncertain time-delay systems, IEEE Transactions on Automatic Control 45(11): 2169-2173.

Ivanesu, D., Dion, J., Dugard, L. and Niculiscu, S.I. (2000). Dynamical compensation for time-delay systems: An LMI approach, International Journal of Robust and Nonlinear Control 10(8): 611-628.

Jiang, X. and Han, Q.-L. (2008). New stability for linear systems with interval time-varying delay, Automatica 44(10): 2680-2685.

Jiang, X. and Han, Q.-L. (2006). Delay-dependent robust stability for uncertain linear systems with interval time-varying delay, Automatica 42(6): 1059-1065. 
Kolmanoskii, V.B. and Myshkis, A.D. (1992). Applied Theory of Functional Differential Equations, Kluwer Academic Publishers, Dordrecht.

Kwon, O.M. and Park, J.H. (2008). Exponential stability for time-delay systems with interval time-varying delays and nonlinear perturbations, Journal of Optimization Theory and Applications 139(2): 277-293.

Kwon, O.M., Lee, S.M. and Park, J.H. (2010). Improved delaydependent exponential stability for uncertain stochastic neural networks with time-varying delays, Physics Letters A 374(10): 1232-1241.

Kim, J.H. (2001). Delay and its time-derivative dependent robust stability of time-delayed linear systems with uncertainty, IEEE Transactions on Automatic Control 46(5): 789-792

Liu, X.W. and Zhang, H.B. (2005). New stability criterion of uncertain systems with time-varying delay, Chaos, Solitons, Fractals 26(5): 1343-1348.

Li, H., Chen, B., Zhou, Q. and Lin, C. (2008). Delay-dependent robust stability for stochastic time-delay systems with polytopic uncertainties, International Journal of Robust and Nonlinear Control 18(15): 1482-1492.

Li, T., Guo, L. and Sun, C. (2007). Robust stability for neural networks with time-varying delays and linear fractional uncertainties, Neurocomputing 71(1-3): 421-427.

Liu, P.-L. (2005). On the stability of neutral-type uncertain systems with multiple time delay, International Journal of Applied Mathematics and Computer Science 15(1):221-229.

Mahmoud, M.S. and Al-Muthairi, N.F. (1994). Quadratic stabilization of continuous time systems with state delay and norm-bounded time-varying uncertainties, IEEE Transactions on Automatic Control 39(10): 2135-2139.

Miyamura, A. and Aihara, K. (2004). Delay-depedent robust stability of uncertain delayed stochastic systems: An LMIbased, Proceedings of the 5th Asian Control Conference, Grand Hyatt-Melbourne, Australia, pp. 449-55.

Ramos, D.C.W. and Peres, P.L.D. (2001). A less conservative LMI condition for the robust stability of discrete-time uncertain systems, Systems \& Control Letters 43(5): 371378 .

Shi, P. and Boukas, E.K. (1997). $H_{\infty}$ control for Markovian jumping linear systems with parametric uncertainty, Journal of Optimization Theory and Applications 95(1): 75-99.

Tian, E., Yue, D. and Gu, Z. (2010). Robust $H_{\infty}$ control for nonlinear systems over network: A piecewise analysis method, Fuzzy Sets and Systems 161(21): 2731-2745.

Xia, Y. and Jia, Y. (2003). Roubst control of state delayed systems with polytopic type uncertainties via parameterdependent Lyapunov functionals, Systems \& Control Letters 50(3): 183-193.

Xia, Y. and Jia, Y. (2002). Robust stability functionals of state delayed systems with polytopic type uncertainties via parameter-dependent Lyapunov functions, International Journal of Control 75(16-17): 1427-1434.
Xu, S., Lam, J., Zou, Y., Zhong, N., Gao, H. and Wang, C. (2004). Robust stabilization for stochastic time-delay systems with polytopic uncertainties, International Conference on Control, Automation, Robotics and Vision, Kunming, China, pp. 1747-1750.

Xue, X. and Qiu, D. (2000). Robust $H_{\infty}$-compensator design for time-delay systems with norm-bounded time-varying uncertainties, IEEE Transactions on Automatic Control 45(7): 1363-1369.

Yue, D., Peng, C. and Tang, G.Y. (2006). Guaranteed cost control of linear systems over networks with state and input quantisations, IEE Proceedings of Control Theory and Applications 153(6): 658-664.

Yan, H.C., Huang, X.H., Zhang, H. and Wang, M. (2009). Delaydependent robust stability criteria of uncertain stochastic systems with time-varying delay, Chaos, Solitons, Fractals 40(4): 1668-1679.

Yue, D. and Han, Q.L. (2005). Delay-dependent exponential stability of stochastic systems with time-varying delay, nonlinearity and Markovian switching, IEEE Transactions on Automatic Control 50(2): 217-222.

Zhang, Y., He, Y. and Wu, M. (2009). Delay-dependent robust stability for uncertain stochastic systems with interval time-varying delay, Acta Automatica Sinica 35(5): 577582

Zhang, Y., He, Y. and Wu, M. (2008). Improved delay-dependent robust stability for uncertain stochastic systems with timevarying delay, Proceedings of the 27th Chinese Control Conference, Kunming, Yunnan, China, pp. 764-768.

Zhou, S., Li, T., Shao, H. and Zheng, W.X. (2006). Output feedback $H_{\infty}$ control for uncertain discrete-time hyperbolic fuzzy sysems, Engineering Applications of Artificial Intelligence 19(5): 487-499.

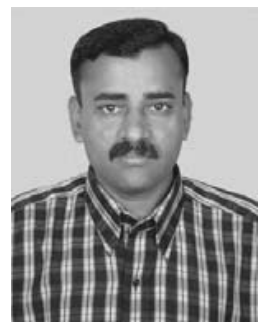

Pagavathigounder Balasubramaniam postgraduated from the Department of Mathematics of Gobi Arts College affiliated to Bharathiar University, Coimbatore, Tamilnadu, India, in 1989. He received an M.Phil. in 1990 and a Ph.D. in 1994 in the field of mathematics from Bharathiar University. Then he served as a lecturer in mathematics at the Kumaraguru College of Technology and Kongu Engineering College for three years. Further, he served as a lecturer in mathematics for four years and a reader in mathematics for five years at Gandhigram Rural University, Gandhigram, Tamilnadu, India. Now he is a professor and the head of the Department of Mathematics, Gandhigram Rural University. He worked as a visiting research professor (2001 and 20052006) for promoting research in the field of control theory and neural networks at Pusan National University, Pusan, South Korea. Recently he has also worked as a visiting professor at the University of Malaya, Malaysia, for six months. He has 18 years of experience in teaching and research. He has published more than 100 journal papers. He is a member of the editorial board of the Journal of Computer Science. Unique recognition was bestowed on him in 2005 through a Tamilnadu Scientist Award (TANSA) in Mathematical Sciences from the Tamilnadu State Council for Science and Technology. His research interests include the areas of control theory, stochastic differential equations, soft computing, neural networks and cryptography. 


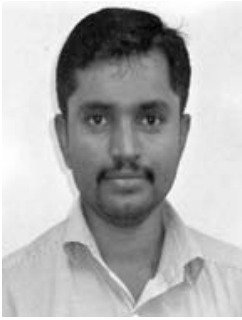

Shanmugam Lakshmanan undergraduated in the field of mathematics (2002-2005) from Government Arts College, Salem. He postgraduated in mathematics from the Sri Ramakrishna Mission Vidyalaya College of Arts and Science affiliated to Bharathiar University, Coimbatore, Tamilnadu, India (2005-2007). In 2008 he was awarded in the field of mathematics by Gandhigram Rural University, Gandhigram, Tamilnadu, India. He was awarded the Ph.D. degree in 2012 by the Department of Mathematics, Gandhigram Rural InstituteDeemed University. His research interest is in the field of qualitative theory of stochastic systems and neural networks.

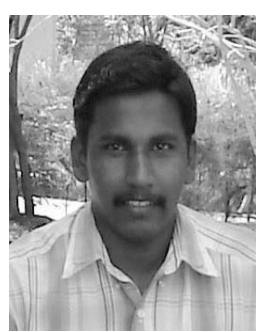

Rajan Rakkiyappan undergraduated in the field of mathematics (1999-2002) from the Sri Ramakrishna Mission Vidyalaya College of Arts and Science. He postgraduated in mathematics from the PSG College of Arts and Science affiliated to Bharathiar University, Coimbatore, Tamilnadu, India (2002-2004). In 2011 he was awarded a Ph.D. by Gandhigram Rural University, Gandhigram, Tamilnadu, India. His research interests include the field of qualitative theory of stochastic and impulsive systems, neural networks and delay differential systems. He has published more than 30 papers in international journals. Now he works as an assistant professor in the Department of Mathematics, Bharathiar University.

Received: 2 August 2010

Revised: 24 January 2011

Re-revised: 10 October 2011 